\title{
A novel hysteroscopic approach for ovarian cancer screening/early diagnosis (Review)
}

\author{
SALVATORE GIZZO $^{1}$, MARCO NOVENTA ${ }^{1}$, MICHELA QUARANTA ${ }^{2}$, AMERIGO VITAGLIANO ${ }^{1}$, \\ CARLO SACCARDI $^{1}$, PIETRO LITTA $^{1}$ and DONATO ANTONA ${ }^{1}$ \\ ${ }^{1}$ Department of Woman and Child Health, University of Padua, I-35128 Padua, Italy; \\ ${ }^{2}$ Department of Obstetrics and Gynecology, Addenbrooke's Hospital, Cambridge CB2 0QQ, UK
}

Received February 22, 2015; Accepted April 12, 2016

DOI: $10.3892 / 01.2016 .5493$

\begin{abstract}
The lethality of epithelial ovarian cancer (EOC) may be due to common misconceptions regarding etiology and the absence of effective screening and early diagnostic tools. Reviews of histopathological surveys performed on the resected fallopian tubes of breast cancer (BRCA) mutation carriers, who underwent risk-reducing salpingo-oophorectomy, unexpectedly revealed the presence of occult carcinomas of the fallopian tubes. This finding prompted studies that demonstrated the most accredited theory of type II EOC development, which suggests that a large proportion of these tumors are derived from the fallopian tube. At present, no diagnostic tools or screening programs have been demonstrated to be effective or cost-effective in improving the outcome of EOC; it is therefore imperative that the scientific community unite its efforts in the identification of a valid screening and/or early diagnostic method for the treatment of this lethal gynecological malignancy. To this end, the present paper proposes a novel tool for the screening/early diagnosis of EOC: The 'Tuba-check'. This novel approach is based on the possibility of acquiring specimens for tubal lumen cytology via hysteroscopy in a minimally-invasive outpatient setting. The present study protocol aimed to validate the technical feasibility and oncological accuracy of the proposed approach, commencing with a cohort of patients with an expected increased oncological risk, including BRCA mutation carriers or those with a gene expression profile of 'BRCA-ness'. If the data collected by the present study protocol validates this approach, the 'Tuba-check' may, in the near future, be extended for the treatment of all women, therefore reducing the number of victims of epithelial ovarian carcinoma.
\end{abstract}

Correspondence to: Professor Salvatore Gizzo, Department of Woman and Child Health, University of Padua, 3 Via Giustiniani, I-35128 Padua, Italy

E-mail: ginecologia_padova@libero.it

Key words: epithelial ovarian cancer, screening tool, early diagnosis, cost-effective strategy, cytological tubal investigation, outpatient setting

\section{Contents}

1. Background

2. Literature review and analysis of evidence

3. Study proposal

4. Expected results and conclusions

\section{Background}

Epithelial ovarian cancer is the most lethal gynecological malignancy worldwide, with a 5-year survival rate of $<30 \%$. Over $75 \%$ of affected women succumb to the disease following combined first line treatment (consisting of surgery and chemotherapy) (1).

Of all the histotypes of ovarian cancer, the most frequent diagnosis in developed countries is high-grade serous carcinoma $(2,3)$, typically characterized by ubiquitous presence of a mutation in the tumor suppressor p53 and, less frequently, by an association with breast cancer (BRCA) 1 and BRCA2 mutations (germline mutations in 13-16\%; somatic mutations in $\sim 6 \%$ of cases) $(4,5)$. Assuming that cancer survival is an indicator of healthcare performance, the lethality of epithelial ovarian cancer (EOC) may be due the common misconceptions regarding etiology and the absence of effective screening and early diagnostic tools (5).

Contrasting with cervical cancer, in which preventive measures and early diagnosis of precancerous lesions has demonstrated to be cost-effective and effective at improving cancer mortality rates $(6,7)$, the current management of EOC remains poor and has been based on existing methods that are directed against established types of cancer, rather than investigating the mechanisms by which EOC develops (8).

Over the previous two decades, proposed EOC screening tools have failed to demonstrate a survival benefit, most likely due to the poor anatomical accessibility of the adnexa, which therefore permits only indirect approaches, including ultrasound evaluation (affected by inter-/intra-operator variability) and serum markers assay (affected by low specificity in precancerous and early lesion detection) (1).

Despite advances in our understanding of the pathogenesis of EOC (8-11), the gap between actual and optimal care in patients at increased risk for EOC emphasizes the urgent 
requirement for identification of a valid screening/early diagnostic strategy. With the aim of collaborating with worldwide scientists and clinicians involved in the search for a novel strategy for the screening and early diagnosis of EOC, following a short and updated summary of the evidence regarding the recent advances and proposals in the field of EOC treatment, the present study proposes an innovative tool to perform screening/early diagnosis in patients at an increased risk of EOC development.

\section{Literature review and analysis of evidence}

Summary of novel evidence concerning the pathogenesis of $E O C$. Currently, the incessant ovulation hypothesis has been almost completely disregarded as an underlying causative mechanism of EOC, as it appears inconsistent with the observation that patients affected by polycystic ovarian syndrome, who demonstrate a decreased number of ovulatory cycles, appear to exhibit an increased risk for the development of ovarian cancer (12). A proposed and accepted paradigm for the development of EOC is the dualistic model, which groups the various types of epithelial ovarian cancer into two broad categories, designated as type I and II $(8,13,14)$.

Type I EOC is comprised of low-grade serous, low-grade endometrioid, mucinous and clear cell carcinomas (15). In general, these tumors are large cystic masses confined to one ovary with a relatively indolent course, and are associated with mutations in Kirsten rat sarcoma viral oncogene homolog, BRAF, phosphatase and tensin homolog, phosphatidylinositol-4,5-bisphosphate 3-kinase catalytic subunit, catenin (cadherin-associated protein) $\beta 1$, AT rich interactive domain $1 \mathrm{~A}$ and protein phosphatase 2 regulatory subunit $\mathrm{A} \alpha$, which perturb signaling pathways (15). These molecular alterations govern the phenotypic expression of a series of transformation events, leading to a stepwise progression from benign, through varying degrees of atypia (borderline tumor), to noninvasive and subsequently invasive low-grade carcinoma (15). Type II tumors are comprised of high-grade serous, high-grade endometrioid, undifferentiated carcinomas and malignant-mixed mesodermal tumors (15). These tumors are biologically aggressive and present at an advanced stage, which contributes to the high rate of mortality (15).

Reviews of histopathological surveys performed on the resected fallopian tubes of BRCA mutation carriers, who underwent risk-reducing salpingo-oophorectomy, unexpectedly revealed the presence of occult carcinomas of the fallopian tubes (a tissue previously thought to rarely exhibit malignancy). This finding prompted studies which demonstrated the most accredited theory of type II EOC development, which suggests that a large portion of these tumors are derived from the fallopian tube (9).

Summary of the recent evidence concerning EOC screening and early diagnosis. Women aged $>40$ years or with a family history of breast and ovarian cancer are commonly considered at the highest risk for development of EOC, and ideally they would be regularly screened for EOC using biomarkers able to detect the disease in the early stages (16).

Despite the substantial efforts put forth by the scientific community and the numerous proposals for non-invasive
EOC screening tools and biomarkers [ranging from International Ovarian Tumor Analysis (IOTA) score assessment by highly skilled sonographers, and the exhaled breath samples test] $(17,18)$, there are currently no known biomarkers or sonographic/radiological approaches that appear sensitive or specific enough to be used as a cost-effective tool for early-stage EOC screening and diagnosis.

Following a systematic review of the literature, it was identified that only a single manuscript proposed a novel approach for the screening/early diagnosis of EOC; this study had a positive rationale as the authors focused the proposal and pilot study on the 'brush cytology of the fallopian tube' (19). Lum et al (19) proposed cytological sampling of the fallopian tube [analogous to the Papanicolaou test (7) for the uterine cervix] to collect endoluminal cells, allowing for discrimination between negative samples and those containing cells suggestive of serous tubal intraepithelial carcinoma, which may be the initial precursor of type II EOC. In the opinion of the authors of the present study, despite the fact that this protocol was unique in its rationale, it may comprehensibly considered only a theoretical proposal due to the presence of several important biases responsible for yielding disappointing results, as well as poor feasibility and reproducibility in a large scale population. The small number of patients $(n=10)$ on which the procedures were performed in addition to the inappropriate eligibility criteria for patient selection made the pilot study useful only for the technical validation of procedure feasibility (all specimens had satisfactory cellularity for interpretation without any complications linked to the procedure), as all cases were interpreted as benign in morphological and immunohistochemical investigations (19). Furthermore, the study failed to demonstrate procedure feasibility due to the erroneous choice of the cervical cytobrush for tubal cytological sampling, which affected the study protocol for at least 3 reasons: i) The diameter and slight flexibility of the cytobrush device does not allow cytological sampling in the infundibulum and fimbriae (in which precursor cells of EOC frequently originate); ii) the mechanical trauma caused by the brush may adversely affect the subsequent histopathological analysis of the tubal epithelium, particularly in situations where the tumor is intraepithelial and the aggregates of the atypical cells are few; and iii) intra-tubal traumatic sampling may irreversibly damage the fallopian tube epithelium, causing a negative impact on functioning (physiologically defined as the ability to convey the products of conception to the uterus). These facts represent a significant problem as the aim of screening is based on maintaining tubal function in patients who have not yet completed their reproductive program (otherwise these patients would be candidates for prophylactic salpingectomy).

As frequently occurs during pioneering research, the analysis of biases affecting previous studies represents a good starting point for the improvement of subsequent protocols. Beginning from the innovative and intuitive study by Lum et al (19), the present study protocol is proposed, with the aim of validating the rationale, usefulness and feasibility of a non-invasive outpatient hysteroscopic approach (known as 'Tuba-check') in the assessment of tubal lumen cell abnormalities. This strategy may potentially be eligible 
as a screening/early diagnostic tool in patients at increased risk of EOC development.

\section{Study proposal}

In order to simplify the experimental model aimed at evaluating the feasibility, accuracy, reproducibility and cost-effectiveness of the proposed novel tool for the screening and early diagnosis of EOC, the presently described protocol intentionally focuses attention on the cohort of patients with BRCA mutations or with a 'BRCA-ness profile' who have been admitted in order to undergo risk-reducing salpingo-oophorectomy due to an increased risk for EOC development (20). The justification for this choice is based on the specific molecular, pathological and clinical features of EOC detected in this cohort of women, as well as the considerable number of prophylactic salpingo-oophorectomies performed with the aim of reducing the risk of EOC development.

The proposed study plans to recruit 100 women with known BRCA1/2 mutations or with a 'BRCA-ness profile', aged 25-75 years and spontaneously adhering to the study protocol following adequate counseling performed in accordance with Italian privacy laws.

Patients will be divided into 2 study groups: Patients aged 35 and older requiring risk-reducing prophylactic salpingo-oophorectomy will be allocated to group A, whereas those aged $<35$ years who choose not to proceed with risk-reducing prophylactic salpingo-oophorectomy for personal choice or because they have not yet completed their reproductive lifespan, will be allocated to group B. In addition, 100 women aged between 25-75 years, with no suspicion of BRCA1/2 mutations or 'BRCA-ness profile' will be recruited, and will be divided into group $\mathrm{C}$ (in the event of salpingo-oophorectomy, during hysterectomy performed for gynecological indications different from adnexal disease) and group D (if salpingo-oophorectomy will not performed).

Patients with a history of any neoplasia, hormonal adjuvant/replacement treatment (including selective estrogen receptor modulators or postmenopausal replacement therapy), adnexal surgery (including treatment for endometriosis, ectopic pregnancy and tubal ligation), absent tubal patency, Müllerian abnormalities, previous cervical surgery (cervical cone or multiple loop excisions and trachelectomy) $(21,22)$ or abnormalities in psychological status will be excluded from the study.

At recruitment, all patients will undergo a gynecological physical examination and pelvic ultrasound scan. In the event of detection of an adnexal mass, adequate characterization will be performed in accordance with IOTA logistic regression 2 recommendations (23).

Furthermore, all patients will undergo cancer antigen 125 (CA125) and human epididymis secretory protein 4 (He4) serum blood sampling with risk of ovarian malignancy algorithm calculation according to hormonal status.

Hysteroscopy using a Bettocchi ${ }^{\circledR} 4.9 \mathrm{~mm}$ Integrated Office hysteroscope (Bettocchi Office Hysteroscopes; Karl Storz GmbH and Co. KG, Tuttlingen, Germany), and uterine cavity distension using normal saline solution with

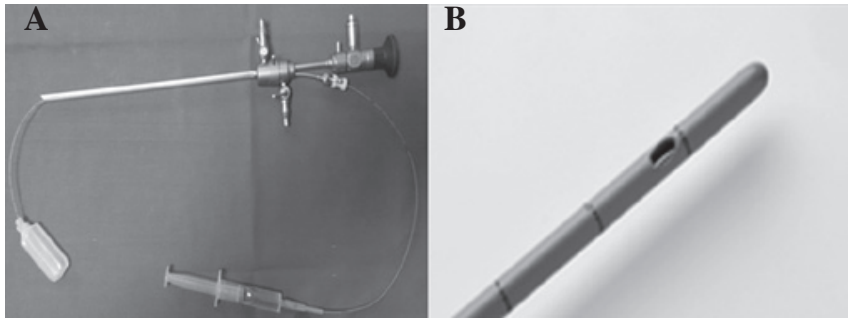

Figure 1. (A) Bettocchi ${ }^{\circledR} 4.9 \mathrm{~mm}$ Integrated Office hysteroscope and (B) atraumatic 4 French sterile ureteric drainage catheter.

an intrauterine pressure set at approximately $35 \mathrm{mmHg}$ (the balance of an irrigation flow of approximately $200 \mathrm{ml} / \mathrm{min}$ and a vacuum of 0.2 bars), will be performed in all eligible women aged 25-75 years old (during the mild follicular phase of the menstrual cycle in the case of premenopausal women).

During the hysteroscopic investigation an atraumatic 4 French sterile ureteric drainage catheter (ColoplastA/S, Humlebaek, Denmark) complete with a cylindrical metal stylet will be introduced through the 4 French sterile ureteric drainage catheter (Fig. 1).

At least $8 \mathrm{~cm}$ of the distal portion of the graduated catheter will be introduced into the fallopian tube lumen (with the aim of reaching the fimbriated extremity); the cylindrical metal stylet will subsequently be removed and following instillation of $5 \mathrm{cc} 9 \% \mathrm{Na} / \mathrm{Cl}$ saline sterile solution, the washing fluid containing the end-luminal cells will be aspirated during catheter retraction. The procedure will be performed bilaterally and the washing fluid from the two fallopian tubes will be sent separately, in sterile tubes, to the cytology department.

Patients in groups A and group C will undergo hysteroscopic tubal washing and aspiration under direct visualization through the umbilical laparoscopic port (necessary for subsequent bilateral laparoscopic adnexectomy). The procedure will therefore be performed under general anesthesia. By contrast, patients admitted to group B and group D will undergo the procedure in an outpatient setting, with routine hysteroscopic tubal sterilization via Essure ${ }^{\circledR}$ (Bayer AG, Leverkusen, Germany) (24).

For all patients, it is planned to collect data concerning general epidemiological features (age, body mass index, ethnicity), family history of breast and ovarian cancer, oral/intrauterine contraceptive use (type and duration), obstetrical history (parity, previous hormonal treatment for infertility care), presence of comorbidities and associated chronic therapy, as well as any previous history of medically treated pelvic inflammatory disease.

Only for patients in group B and group D (outpatient setting) will data regarding perceived pain during the hysteroscopic procedure be collected, through a numeric rating scale self-administered score (prior to and following the procedure), and patient satisfaction at the end of procedure will be assessed through a visual analogue scale with a value ranging from 0 (no satisfaction) to 10 (complete satisfaction).

For all patients data on the following will also be collected: Procedure failure due to technical difficulties, number of non-diagnostic samples due to poor cellularity, 
number of intraoperative complications, including bleeding or tubal perforation, and number of postoperative complications, including refractory pelvic pain or fever.

Additionally, data regarding preoperative ultrasound investigation and CA125/He4 serum assays will be collected. Finally, it is planned to collect information in cases in which cytological anomalies are detected and, exclusively for patients in group A and group C, data regarding histological examination of the removed adnexa.

Study aims. The primary aim of the discussed study protocol is to compare patients in group A and group C with those included in group B and group D, in terms of perioperative pain, patient satisfaction and complication rate in order to validate the safety and technical feasibility of the procedure in an outpatient setting.

The groups will also be compared in terms of cellularity of the collected washing fluid, and whether this fluid is sufficient to allow cytological diagnosis in order to validate the potential applicability and rationale of the proposed procedure.

The secondary aim of the discussed protocol is to correlate the cytological investigation results from group $\mathrm{A}$ and group $\mathrm{C}$ with definitive histology in order to validate the accuracy of cytological tubal washing in the detection or exclusion of cases with or without cellular abnormalities.

The tertiary aim of the discussed protocol is to correlate preoperative epidemiological characteristics, ultrasound features and serum biomarker values with cytological and histological (when performed) diagnoses in order to identify associations, if these exist.

\section{Expected results and conclusions}

As current evidence demonstrates that no diagnostic tools or screening programs are effective or cost-effective at improving the outcome of EOC, it is therefore imperative that the scientific community unite its efforts in the search of a valid screening and/or early diagnostic method for the treatment of this lethal gynecological malignancy.

Scientific literature continues to validate hysteroscopy as a safe routine diagnostic procedure characterized by a short learning curve for a tubal approach (as in female sterilization), since upon performing $<50$ procedures under tutor support, generally clinicians acquire the skills required to perform the procedure independently (24). It is therefore possible that the hysteroscopy approach to the fallopian tube may be considered to be the most simple, minimally-invasive and large scale reproducible tool available. Technical difficulties are expected to arise with the protocol discussed in this paper, however a close collaboration with biomedical engineers should allow potential obstacles to be overcome. For the above-mentioned reasons, the validation of technical feasibility has been set as the primary endpoint of the presently discussed study protocol, and determination of the accuracy in predicting cell abnormalities has been set as the secondary endpoint.

If the data collected by the currently summarized study protocol will validate this approach, it is likely that in the near future the 'Tuba-check' can be improved and made available to the entire female population for the treatment of EOC, therefore reducing the number of mortalities.

\section{References}

1. Coleman MP, Forman D, Bryant H, Butler J, Rachet B, Maringe C, Nur U, Tracey E, Coory M, Hatcher J, et al; ICBP Module 1 Working Group: Cancer survival in Australia, Canada, Denmark, Norway, Sweden, and the UK, 1995-2007 (the International Cancer Benchmarking Partnership): An analysis of population-based cancer registry data. Lancet 377 : 127-138, 2011.

2. Siegel R, Naishadham D and Jemal A: Cancer statistics, 2013. CA Cancer J Clin 63: 11-30, 2013.

3. George SH and Shaw P: BRCA and early events in the development of serous ovarian cancer. Front Oncol 4: 5, 2014.

4. Kuhn E, Kurman RJ, Vang R, Sehdev AS, Han G, Soslow R, Wang TL and Shih IeM: TP53 mutations in serous tubal intraepithelial carcinoma and concurrent pelvic high-grade serous carcinoma - evidence supporting the clonal relationship of the two lesions. J Pathol 226: 421-426, 2012.

5. Cancer Genome Atlas Research Network: Integrated genomic analyses of ovarian carcinoma. Nature 474: 609-615, 2011.

6. Gizzo S, Noventa M and Nardelli GB: Gardasil administration to hr-HPV-positive women and their partners. Trends Pharmacol Sci 34: 479-480, 2013.

7. Saccardi C, Gizzo S, Noventa M, Anis O, Di Gangi S, Patrelli TS, D'Antona D and Nardelli GB: High risk human papillomavirus DNA test: Could it be useful in low-grade cervical lesion triage? Five-year follow-up. Reprod Sci 21: 198-203, 2014.

8. Kurman RJ: Origin and molecular pathogenesis of ovarian high-grade serous carcinoma. Ann Oncol 24 (Suppl 10): $\mathrm{x} 16-\mathrm{x} 21,2013$.

9. Erickson BK, Conner MG and Landen CN Jr: The role of the fallopian tube in the origin of ovarian cancer. Am J Obstet Gynecol 209: 409-414, 2013.

10. Koshiyama M, Matsumura N and Konishi I: Recent concepts of ovarian carcinogenesis: Type I and type II. Biomed Res Int 2014: 934261, 2014.

11. Li J, Abushahin N, Pang S, Xiang L, Chambers SK, Fadare O, Kong B and Zheng W: Tubal origin of 'ovarian' low-grade serous carcinoma. Mod Pathol 24: 1488-1499, 2011.

12. Schildkraut JM, Schwingl PJ, Bastos E, Evanoff A and Hughes C: Epithelial ovarian cancer risk among women with polycystic ovary syndrome. Obstet Gynecol 88: 554-559, 1996.

13. Shih IeM and Kurman RJ: Ovarian tumorigenesis: A proposed model based on morphological and molecular genetic analysis. Am J Pathol 164: 1511-1518, 2004.

14. Kurman RJ and Shih IeM: Molecular pathogenesis and extraovarian origin of epithelial ovarian cancer - shifting the paradigm. Hum Pathol 42: 918-931, 2011.

15. Gizzo S,BerrettaR,Di Gangi S, Guido M,Zanni GC,Franceschetti I, Quaranta M, Plebani M, Nardelli GB and Patrelli TS: Borderline ovarian tumors and diagnostic dilemma of intraoperative diagnosis: Could preoperative He4 assay and ROMA score assessment increase the frozen section accuracy? A multicenter case-control study. Biomed Res Int 2014: 803598, 2014.

16. Lawrenson K, Mhawech-Fauceglia P, Worthington J, Spindler TJ, O'Brien D, Lee JM, Spain G, Sharifian M, Wang G, Darcy KM, et al: Identification of novel candidate biomarkers of epithelial ovarian cancer by profiling the secretomes of three-dimensional genetic models of ovarian carcinogenesis. Int J Cancer 137: 1806-1817, 2015.

17. Testa A, Kaijser J, Wynants L, Fischerova D, Van Holsbeke C, Franchi D, Savelli L, Epstein E, Czekierdowski A, Guerriero S, et al: Strategies to diagnose ovarian cancer: New evidence from phase 3 of the multicentre international IOTA study. Br J Cancer 111: 680-688, 2014.

18. Amal H, Shi DY, Ionescu R, Zhang W, Hua QL, Pan YY, Tao L, Liu $\mathrm{H}$ and Haick H: Assessment of ovarian cancer conditions from exhaled breath. Int J Cancer 136: E614-E622, 2015.

19. Lum D, Guido R, Rodriguez E, Lee T, Mansuria S, D'Ambrosio L and Austin RM: Brush cytology of the fallopian tube and implications in ovarian cancer screening. J Minim Invasive Gynecol 21: 851-856, 2014.

20. Gizzo S, Noventa M, Saccardi C, Litta P, D'Antona D and Nardelli GB: Proposal on Raloxifene use after prophylactic salpingo-oophorectomy in BRCA1-2: Hypothesis and rationale. Eur J Cancer Prev 23: 514-515, 2014. 
21. Gizzo S, Ancona E, Saccardi C, Patrelli TS, Berretta R, Anis O, Noventa M, Bertocco A, Fagherazzi S, Longone M, et al: Radical trachelectomy: The first step of fertility preservation in young women with cervical cancer (Review). Oncol Rep 30: 2545-2554, 2013.

22. Berretta R, Gizzo S, Dall'Asta A, Mazzone E, Monica M, Franchi L, Peri F, Patrelli TS and Bacchi Modena A: Risk of preterm delivery associated with prior treatment of cervical precancerous lesion according to the depth of the cone. Dis Markers 35: 721-726, 2013.
23. Kaijser J, Sayasneh A, Van Hoorde K, Ghaem-Maghami S, Bourne T, Timmerman D and Van Calster B: Presurgical diagnosis of adnexal tumours using mathematical models and scoring systems: A systematic review and meta-analysis. Hum Reprod Update 20: 449-462, 2014.

24. Gizzo S, Bertocco A, Saccardi C, Di Gangi S, Litta PS, D'antona D and Nardelli GB: Female sterilization: Update on clinical efficacy, side effects and contraindications. Minim Invasive Ther Allied Technol 23: 261-270, 2014. 